\title{
Topological properties of random wireless networks
}

\author{
SRIKANTH K IYER ${ }^{1}$ and D MANJUNATH ${ }^{2}$ \\ ${ }^{1}$ Department of Mathematics, Indian Institute of Science, Bangalore 560 012, India \\ ${ }^{2}$ Department of Electrical Engineering, Indian Institute of Technology - Bombay, \\ Powai, Mumbai 400076 India \\ e-mail: skiyer@math.iisc.ernet.in; dmanju@ee.iitb.ac.in
}

\begin{abstract}
Wireless networks in which the node locations are random are best modelled as random geometric graphs (RGGs). In addition to their extensive application in the modelling of wireless networks, RGGs find many new applications and are being studied in their own right. In this paper we first provide a brief introduction to the issues of interest in random wireless networks. We then discuss some recent results for one-dimensional networks with the nodes distributed uniformly in $(0, z)$. We then discuss some asymptotic results for networks in higher dimensions when the nodes are distributed in a finite volume. Finally we discuss some recent generalisations in considering non uniform transmission ranges and non uniform node distributions. An annotated bibliography of some of the recent literature is also provided.
\end{abstract}

Keywords. Wireless networks; geometric random graphs; topology properties.

\section{Introduction}

Wireless networks come in many flavours - the by now well-established cellular networks, 'Wi-Fi' networks, ad hoc networks and, more recently, sensor networks. In cellular networks and in one version of the 'Wi-Fi' network, all communication between nodes is through a central 'base station' or 'access-point'. Such networks are 'infrastructure based' networks indicating that a physical infrastructure needs to be put in place before nodes can communicate. Ad hoc and sensor networks are multihop networks in which the paths taken by data flows could involve one or more intermediate nodes that relay the data in the direction of the destination of the flow over wireless links. In this paper, we concentrate on multihop wireless networks. By mapping nodes of the wireless network to vertices and communicating links to edges, the communication paths of the wireless network can be represented by a graph. The representation of the infrastructure wireless networks is a star graph while that of a multihop network is an arbitrary graph.

An important aspect of wireless networks is node mobility, i.e., node locations change randomly and at random times. In sensor networks, a typical assumption is that the sensors are deployed randomly in the operational area. Communicating links in this network depend on the relative locations of the nodes. Since the node locations are random, we have a random 
network. Our interest in this paper is to explore the tools to study the statistical properties of the topology of such random networks. To do that we construct a random graph model for wireless networks when the nodes are placed randomly in their operational area.

Let the nodes of the wireless network be randomly distributed in $d$-dimensional space ( $d=1,2,3$ have direct relevance in the context of wireless networks). The probability with which a signal from a wireless transmitter can be correctly decoded by a receiver depends on the ratio of the received signal to the sum of interference and noise (SINR) at the receiver. The received power decays according to a power law with the distance from the transmitter, i.e., as $\left(1 /\right.$ distance $\left.^{\alpha}\right)$, with $\alpha>2$ being typical. Thus the existence of a communication channel between two wireless nodes and its quality depends on the transmission power, relative locations of the transmitter and the receiver and the radio environment in which the signal has to propagate. We make the reasonable (and standard) assumption that receivers have strict SINR requirements, i.e., if the SINR is above a threshold, then the decoding error is acceptable and we assume that reliable communication between the nodes is possible, otherwise no communication is possible. This assumption translates to the following condition. When node $i$ at location $X_{i}$ is transmitting, node $j$ (at location $X_{j}$ ) can decode the transmission if and only if the distance between the two nodes is less than $w_{i, j} . w_{i, j}$ is determined by SINR threshold requirement of node $j$ and the transmission power of node $i$. Clearly, depending upon the transmission power of the nodes and the distances between them, only a small subset of the nodes can communicate directly over one-hop paths. Those that cannot communicate directly send their data over multihop paths.

For any realisation of the node distribution in the operational area, we can obtain a graph, denoted by $G_{w}:=(V, E)$, that represents the connectivity in the network. $G_{w}$ is obtained as follows. Each node in the network corresponds to a vertex in $V$. Edge $e_{i} \in E,\left(e_{i}:=\left(i_{1}, i_{2}\right)\right)$ iff the transmission from node $i_{1}$ can be decoded at node $i_{2}$, i.e., iff $\left\|X_{i_{1}}-X_{i_{2}}\right\|<w_{i_{1}, i_{2}}$. Here $\|\cdot\|$ denotes the Euclidean distance between nodes $i_{1}$ and $i_{2}\left(l_{2}\right.$ norm; other norms may also be used) and $w_{i, j}$ is called the 'edge distance' or 'cutoff' for node pair $(i, j)$. As we said earlier, $w_{i_{1}, i_{2}}$ depends on the transmission power of $i_{1}$ and the receiver sensitivity of $i_{2}$. We can assume symmetry in the one hop links (i.e., existence of edge $\left(i_{1}, i_{2}\right)$ implies the existence of edge $\left.\left(i_{2}, i_{1}\right)\right)$, and consider only an undirected graph $G_{w}$. Randomness of the coordinates of the node in the distribution area introduces randomness in the graph.

Till recently, two well-known random graph models have been used to model random networks - the $G_{p}$ and the $G_{k}$ random graphs that are constructed as follows. In the $G_{p}$ random graph of $N$ vertices, edge $e_{k}=\left(v_{k_{1}}, v_{k_{2}}\right)$ occurs with probability $p$ independent of other edges. The $G_{k}$ random graph of $N$ vertices is constructed as follows. $M\left(:=\left(\begin{array}{c}N \\ 2\end{array}\right)\right)$ edges are possible in $N$ node network. To construct a graph of $k$ edges, we randomly choose $k$ edges from the $M$ possible, i.e., each of $\left(\begin{array}{c}M \\ k\end{array}\right)$ graphs are equally likely. These graphs are called Erdos-Renyi random graphs (see Bollobas 1985, for a detailed discussion of these graphs) and are not directly applicable for modelling of random wireless networks. Clearly, the $G_{p}$ random graph is not the same as $G_{w}$ because edges are not independent in $G_{w} ; G_{k}$ is also not the same as $G_{w}$ because the number of edges in $G_{w}$ is not predetermined. $G_{w}$ are called random geometric graphs (RGGs) because the graph is derived from geometric realisations. In this paper we study the topological properties of random geometric graphs, $G_{w}$.

Three classes of properties are of interest in the context of wireless networks - (1) topological properties, (2) communications properties and (3) sensing properties. The topological properties of interest are essentially the same as that for the Erdos-Renyi graphs. Given $w_{i, j}$, the probability that the network is connected is an important example. Conversely, what classes of $w_{i, j}$ would make $G_{w}$ connected with a specified probability? Similar questions 
could be asked about the minimum, maximum and average vertex degrees. Other properties could be chromaticity and the number of components in $G_{w}$. An early survey of results can be obtained from Diaz et al (1998). In the context of sensor networks, each node is expected to 'sense' a certain area around it, say $a_{i}$ around the point where node $i$ is deployed. If the nodes are deployed randomly, there could be some areas that are not covered by any sensor. Our interest could be in finding the properties of such areas for a given $a_{i}$ or in determining the properties of $a_{i}$ to achieve a coverage objective.

In studying the communication properties, we need to specify the communication model. For example, we could have $N / 2$ randomly selected pairs communicating via multihop paths. Alternatively, we could have a central node collecting data from all the other nodes and/or disseminate data to all the other nodes. Essentially the objective is to compute a 'sensing function' in a distributed manner, e.g., the minimum, maximum or average temperature in the area. This requires a certain pattern of information exchange. We also need to specify the communication constraints. Typically, this is done by specifying the edges along which communication can occur simultaneously. A classical constraint in wireless networks is that two nodes cannot transmit simultaneously if they are one-hop or two-hop neighbours. Alternatively, we could only restrict that neighbours of a receiving node should not be transmitting simultaneously. An important issue is the maximum rate at which information could be exchanged in the network according to the specified model and constraints.

Sensor networks collect sample environmental data and perform distributed processing on the samples. A performance measure of interest is the rate at which this function can be computed over an underlying random network subject to constraints like, for example, energy consumption. In this case we could be interested in the time and energy complexity in the execution distributed function (Giridhar \& Kumar 2005; Khude et al 2005). A canonical function is the localisation where the position of a target, a source of interest or even the nodes themselves needs to be obtained. Here constraints on the errors in the estimate may also be specified. A sensor network could also track the function in time where a continuous estimate is to be obtained using estimates from discrete instants.

The list of properties that we mention above are clearly not exhaustive and only illustrative. In this paper we will concentrate on only the topological properties of random networks. An excellent introduction to random geometric graphs is available in (Penrose 2003). This paper has the following two primary objectives.

- Provide a survey of the results that have not been considered by Penrose (2003). Specifically, we consider finite graphs, non uniform distribution of nodes and also non uniform cutoffs.

- Provide new proofs to previously known results for one-dimensional networks.

To complete the picture, we also provide a tutorial on the proof techniques of Penrose (2003) and summarise some important results there. Except for $\S 4$. 1 , we assume that $w_{i, j}=w_{j, i}=r$ for all $i, j$.

The rest of the paper is organised as follows. In the next section we discuss one-dimensional networks in which the nodes are distributed uniformly in $[0, z]$. This is illustrative of many of the issues in the analysis of $G_{w}$. The exact probability that a network of a finite number of nodes is connected is first derived. New proofs are provided for some strong law results on one-dimensional networks. In $\S 3$ we consider graphs in higher dimensions. Results for finite graphs are not available in higher dimensions and only asymptotic results are described. We first define two possible limiting regimes as the number of nodes $n \rightarrow \infty$. In this paper we describe only one of these - the connectivity regime. The use of Poissonisation and 
de-Poissonisation in the proofs of the asymptotic results are then explained. We describe a weak convergence result for the number of graphs isomorphic to a given connected graph on $k$ vertices. We then describe the derivation of some strong law results for the maximum vertex degree, chromaticity and minimum vertex degree under the connectivity limiting regime. Sections 2 and 3 assume that $w_{i, j}=r$ for all $1 \leq i<j \leq n$. In $\S 4$, we first generalise to arbitrary $w_{i, j}$ and obtain some properties under the $l_{\infty}$ norm of measuring the distances. We then describe some results when the node locations are i.i.d. exponential in $(0, \infty)$. Finally, in $\S 5$ we provide an annotated bibliography of the literature on the topological analysis of random wireless networks and random geometric graphs.

\section{One-dimensional networks}

We first summarise the connectivity analysis of a finite one-dimensional network from Desai \& Manjunath (2002). Distribute $n$ nodes uniformly in $[0, z]$. Let $X_{i}$ denote the location of node $i$. A simple motivation for such a network is in constructing a intrusion detection sensor network with nodes deployed randomly along a border. Assume $w(i, j)=r$ for $1 \leq i<j \leq n$. Let $N=\left[X_{1}, X_{2}, \ldots, X_{n}\right]$ denote a realisation of the network and $P_{c}(n, z, r)$ denote the probability that the network $N$ forms a connected graph. Let $X_{(i)}$ be the $i$ th node from the origin with $i=1, \ldots, n$, i.e., $X_{(1)}<X_{(2)}<\cdots,<X_{(n)}$. Let $\hat{N}:=\left[X_{(1)}, X_{(2)}, \ldots, X_{(n)}\right]$ and $X_{(0)}=0 . N$ is connected iff $X_{(i+1)}-X_{(i)}<r$ for $i=1, \ldots,(n-1) . P_{c}(n, z, r)$ is obtained as follows.

The set of all realizable $N$ is contained in the $n$-dimensional polytope, $A_{n}$, defined by $0 \leq X_{(1)} \leq X_{(2)} \leq \cdots, \leq X_{(n)} \leq z$. The set of connected networks is contained in the $n$ dimensional polytope, $A_{c}(n, z, r)$, defined by $X_{(i+1)}-X_{(i)}<r$ for $i=1, \ldots,(n-1)$. Let $V_{n}(z)$ and $V_{c}(n, z, r)$ be the volumes of the polytopes $A_{n}$ and $A_{c}(n, z, r)$ respectively. Since the nodes are uniformly distributed in $[0, z], P_{c}(n, z, r)=V_{c}(n, z, r) / V_{n}(z)$.

Define $Y=\left[Y_{0}, \ldots, Y_{n-1}\right]$ where $Y_{i}=X_{(i+1)}-X_{(i)}, i=0, \ldots n-1$. Let $U(n, z)$ be the volume of the set

$$
\left\{Y_{0}, Y_{1}, \ldots Y_{n-1}: Y_{i} \geq 0 \text { for } i \geq 0, \sum_{0}^{n-1} Y_{i} \leq z\right\},
$$

and $U_{c}(n, z, r)$ be the volume of the set

$$
\left\{Y_{0}, Y_{1}, \ldots Y_{n-1}: Y_{i} \geq 0 \text { for } i \geq 0, Y_{i} \leq r \text { for } i>0, \sum_{0}^{n-1} Y_{i} \leq z\right\} .
$$

Since $\hat{N}$ and $Y$ are related by a linear invertible transformation, $U_{n}(z)=K V_{n}(z)$ and $U_{c}(n, z, r)=K V_{c}(n, z, r)$ for some constant $K>0$ and we can write

$$
P_{c}(n, z, r)=\left\{U_{c}(n, z, r)\right\} /\left\{U_{n}(z)\right\} .
$$

It can be shown that $U_{n}(z)$ and $U_{c}(n, z, r)$ are given by the following relations.

$$
\begin{aligned}
U_{n}(z) & =\int_{0}^{z} U_{n-1}(z-t) \mathrm{d} t=z^{n} / n !, \\
U_{c}(n, z, r) & =\int_{0}^{r} U_{c}(n-1, z-t, r) \mathrm{d} t .
\end{aligned}
$$




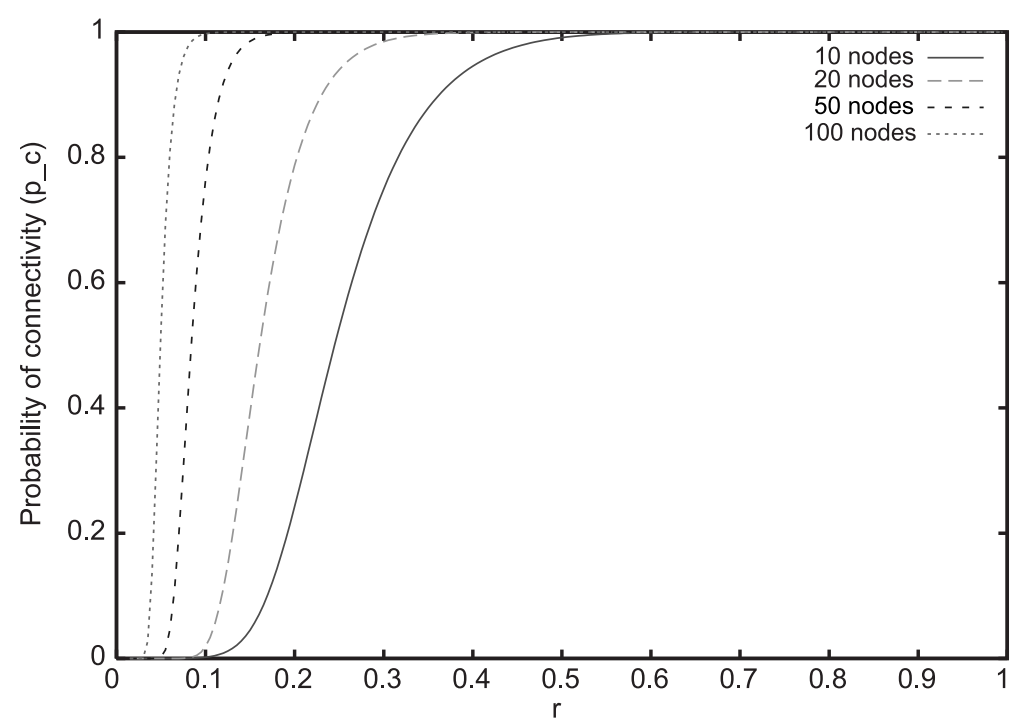

Figure 1. Probability of the network being connected as a function of $r$, the transmission radius. We have used $z=1$ and show $P_{c}(n, 1, r)$ for different $n$.

After some algebra, we can show that

$$
U_{c}(n, z, r)=\sum_{k=0}^{n-1}\left(\begin{array}{c}
n-1 \\
k
\end{array}\right) \frac{(-1)^{k}(z-k r)^{n} u(z-k r)}{n !},
$$

and hence

$$
P_{c}(n, z, r)=\sum_{k=0}^{n-1}\left(\begin{array}{c}
n-1 \\
k
\end{array}\right)(-1)^{k} \frac{(z-k r)^{n}}{z^{n}} u(z-k r) .
$$

Here $u(x)$ is the unit step function or the Heaviside function.

Equation (1) can be derived as a special case of the probability of there being $m$ components in $G_{w}$. Godehardt \& Jaworsky (1996) has shown that the probability of there being $k$ components is given by

$$
\sum_{k=m-1}^{n-1}\left(\begin{array}{c}
n-1 \\
k
\end{array}\right)\left(\begin{array}{c}
k \\
m-1
\end{array}\right)(-1)^{k+m-1}(1-k r)^{n}
$$

For $z=1.0$, figure 1 shows a plot of $P_{c}(n, r, z)$ as a function of $r$ for different values of $n$. We see that as $r$ increases, the transition from being disconnected with high probability to being connected with high probability becomes sharper as $n$ increases. Of course, we also see that the $r$ required to make the network connected with high probability decreases with increasing $n$. We next ask if there is 'thresholding' behaviour of the connectivity property, i.e., is there an $r_{n}$ which is a 'critical $r$ ' to make the network connected. Also, if such behaviour exists, then what is the threshold $r_{n}$ ? We first make the notion of thresholding more precise.

If a graph $G$ possesses the property $\mathcal{P}$, we write $G \in \mathcal{P}$. A graph property $\mathcal{P}$ is a monotone increasing property if for any two graphs $G_{1}=\left(V, E_{1}\right)$ and $G_{2}=\left(V, E_{2}\right), E_{1} \subset E_{2}$ and 
$G_{1} \in \mathcal{P}$ implies $G_{2} \in \mathcal{P}$. A monotone increasing property continues to hold when edges are added to a graph, For random geometric graphs and a given set of node locations $X_{i}$, $i=1,2, \ldots$, increasing $r$ only adds new edges to the graph. Hence, a monotone increasing property is preserved by increasing $r$ in a given realisation of the node distribution. The complement of any monotone increasing property is a monotone decreasing property. A monotone decreasing property is unaltered by the deletion of edges, and hence by decreasing of $r$. Connectivity of a graph $G$ is a monotone increasing property and so is the property that the maximum vertex degree is greater than $k$. The property that the minimum vertex degree is less than $k$ is a monotone decreasing property. McColm and Goel et al (2004) calls a monotone increasing property an upwards closed property and a monotone decreasing property, a downwards closed property.

Consider a sequence $G_{n}$ of random geometric graphs with graph $G_{n}$ obtained using a cutoff $r_{n}$, i.e., $w_{i, j}=r_{n}$ for all $i, j$. A sequence $t_{n}$ of cutoffs is a weak threshold function for a monotone property $\mathcal{P}$ iff $r_{n} / t_{n} \rightarrow \infty$ implies that $\operatorname{Pr}\left(G_{n} \in \mathcal{P}\right) \rightarrow 1$ and $r_{n} / t_{n} \rightarrow 0$ implies $\operatorname{Pr}\left(G_{n} \in \mathcal{P}\right) \rightarrow 0$. We can also define a strong threshold function $t_{n}$ for property $\mathcal{P}$ as follows: if for all $0<\epsilon<1, r_{n}=(1-\epsilon) t_{n}$ implies $\operatorname{Pr}\left(G_{n} \in \mathcal{P}\right) \rightarrow 0$ and $r_{n}=(1+\epsilon) t_{n}$ implies $\operatorname{Pr}\left(G_{n} \in \mathcal{P}\right) \rightarrow 1$. Another definition of a threshold is from Friedgut \& Kalal (1996). For a monotone increasing property $\mathcal{P}$, let $\epsilon$ be such that $0<\epsilon<1 / 2$. Let $r(n, \epsilon)=\inf \{r>0$ : $\left.\operatorname{Pr}\left(G_{n} \in \mathcal{P}\right)>\epsilon\right\}$. Define the threshold width $\delta(n, \epsilon):=r(n, 1-\epsilon)-r(n, \epsilon)$. Property $\mathcal{P}$ is said to have a sharp threshold if $\delta(n, \epsilon)=o(1)$ for all $0<\epsilon<1 / 2$.

In the following we develop new proofs for the asymptotic analysis of connectivity and nearest neighbour distance.

\subsection{Asymptotic analysis}

To prove asymptotic results we need to consider a sequence $G_{n}$ with $G_{n}$ being an RGG of $n$ nodes. $G_{n}\left(r_{n}\right)$ will be the $n$-node RGG with $w_{i, j}=r_{n}$. Let $c_{n}$ be the smallest edge distance required to make the graph $G_{n}$ connected. $c_{n}$ is called the connectivity distance and is defined as

$$
c_{n}:=\inf \left\{r>0: G_{n}(r) \text { is connected }\right\},
$$

Similarly, let $d_{n}$ be the smallest edge distance for which the minimum vertex degree is 1 . $d_{n}$ is called the largest nearest neighbour distance. and is defined as

$$
\begin{aligned}
D_{i} & = \begin{cases}\min \left(\left|X_{(i)}-X_{(i-1)}\right|,\left|X_{(i+1)}-X_{(i)}\right|\right), & \text { for } i=2, \ldots, n-1, \\
\left|X_{(n)}-X_{(n-1)}\right|, & \text { for } i=n,\end{cases} \\
d_{n} & :=\max _{2 \leq i \leq n}\left\{D_{i}\right\} .
\end{aligned}
$$

Asymptotic results for $c_{n}$ and $d_{n}$ are obtained using a graph $G_{n}^{*}\left(r_{n}\right)$ which is constructed using independent exponential spacings as follows. Let $Y_{n, 0}, Y_{n, 1}, \ldots, Y_{n, n}$ be i.i.d. exponential random variables with parameter $n$. The vertex set of the graph $G_{n}^{*}\left(r_{n}\right)$ is the set of points $V_{n}^{*}=\left\{Y_{j}=\sum_{i=0}^{j-1} Y_{n i}\right\}_{j=1}^{n}$. The edge set of this graph, $E_{n}^{*}=\left\{\left(Y_{i}, Y_{j}\right):\left|Y_{i}-Y_{j}\right|<r_{n}\right\}$.

The following lemma of McColm (2004) provides the connection between the graphs $G_{n}$ and $G_{n}^{*}$.

Lemma 1. Let $\Theta$ be an monotone increasing property and let $r_{n}, \alpha, \epsilon>0$. There is a $N>0$, such that for all $n \geq N$, the following holds: 
1. If $\operatorname{Pr}\left(G_{n}^{*}\left(r_{n}\right) \in \Theta\right)<\alpha$, then $\operatorname{Pr}\left(G_{n}\left((1-\epsilon) r_{n}\right) \in \Theta\right)<\alpha /(1-\alpha)$.

2. If $\operatorname{Pr}\left(G_{n}\left(r_{n}\right) \in \Theta\right)<\alpha$, then $\operatorname{Pr}\left(G_{n}^{*}\left((1-\epsilon) r_{n}\right) \in \Theta\right)<2 \alpha$.

3. If $\operatorname{Pr}\left(G_{n}^{*}\left(r_{n}\right) \in \Theta\right)>1-\alpha$, then $\operatorname{Pr}\left(G_{n}\left((1+\epsilon) r_{n}\right) \in \Theta\right)>(1-2 \alpha) /(1-\alpha)$.

4. If $\operatorname{Pr}\left(G_{n}\left(r_{n}\right) \in \Theta\right)>1-\alpha$, then $\operatorname{Pr}\left(G_{n}^{*}\left((1+\epsilon) r_{n}\right) \in \Theta\right)>1-2 \alpha$.

Simply put, the above lemma states that if the graph $G_{n}$ has a monotone increasing property with asymptotically small probability, then the same is true for the graph $G_{n}^{*}$ and vice-versa. Thus, the graphs $G_{n}$ and $G_{n}^{*}$ have the same thresholds. Further, if $\operatorname{Pr}\left(G_{n}\left(r_{n}\right) \in \Theta\right)$ is bounded by a sequence $\alpha_{n}$ which is summable, then the same is true for $\operatorname{Pr}\left(G_{n}^{*}\left(r_{n}\right) \in \Theta\right)$ and viceversa. Lastly, as a corollary, the above conclusions can be stated for monotone decreasing property as well. Thus this lemma allows us to study $G_{n}$ through $G_{n}^{*}$.

A simple application of lemma 1 is to derive the connectivity threshold for the graph $G_{n}(r)$. Let $P_{c}\left(r_{n}\right)$ and $P_{c}^{*}\left(r_{n}\right)$ be the respective probabilities that the graphs $G_{n}\left(r_{n}\right)$ and $G_{n}^{*}\left(r_{n}\right)$ are connected.

Theorem 1. $r_{n}=\frac{\log n}{n}$ is a strong threshold for connectivity of the graph $G_{n}$.

Proof. Since both graphs have the same connectivity thresholds, we consider $P_{c}^{*}\left(r_{n}\right)$.

$$
P_{c}^{*}\left(r_{n}\right)=\operatorname{Pr}\left(Y_{n 1}<r_{n}, \ldots, Y_{n(n-1)}<r_{n}\right)=\left(1-e^{-n r_{n}}\right)^{n} .
$$

It is easy to see that if we take $r_{n}=\left(\log n+d_{n}\right) / n$, such that $d_{n} \rightarrow d \in[-\infty, \infty]$, then $P_{c}\left(r_{n}\right) \rightarrow \exp (-\exp (-d))$. Thus $P_{c}\left(r_{n}\right) \rightarrow 0$, if and only if $d=-\infty$ and $P_{c}\left(r_{n}\right) \rightarrow 1$, if and only if $d=\infty$. Thus $\log n / n$ is a weak threshold for connectivity of $G_{n}^{*}$. Taking $d_{n}=(1+\epsilon) \log n$, we see that $(\log n / n)$ is also a strong threshold for $G_{n}^{*}$.

We now consider the asymptotics for the number of components or the number of clusters in the graph $G_{n}$. Let $r_{n}$ be a sequence of edge distances and let $m_{n}=n \exp \left(-n r_{n}\right)$. Let $K_{n}$ (respectively, $\left.K_{n}^{*}\right)$ be the number of components or clusters at level $r_{n}$ in the graph $G_{n}\left(r_{n}\right)$ (respectively $G_{n}^{*}\left(r_{n}\right)$ ).

Theorem 2. (i) If $m_{n} \rightarrow \mu<\infty$, then $K_{n}$ converges in distribution to a Poisson random variable with mean $\mu$.

(ii) If $m_{n} \rightarrow \infty$, then $\left(K_{n}-m_{n}\right) / \sqrt{m_{n}}$ converges in distribution to a standard normal random variable.

Proof. (i) $K_{n}^{*}=\sum_{i=1}^{n-1} I_{\left\{Y_{n i}>r_{n}\right\}}$. Again we prove the results for the graph $G_{n}^{*}$, since the event $\left\{K_{n} \leq x\right\}$ is upwards closed. The moment generating function (MGF) of $K_{n}^{*}$ is given by

$$
\begin{aligned}
\mathrm{E}\left(e^{t K_{n}^{*}}\right) & =\left[1+\left(e^{t}-1\right) e^{-n r_{n}}\right]^{n} \\
& =\exp \left(n \exp \left(-n r_{n}\right)\left(e^{t}-1\right)+o\left(\frac{1}{n}\right)\right) \rightarrow \exp \left(\mu\left(e^{t}-1\right)\right) .
\end{aligned}
$$

(ii)

$$
\begin{aligned}
\mathrm{E}\left(e^{t\left(K_{n}^{*}-m_{n}\right) / \sqrt{m_{n}}}\right) & =e^{-t \sqrt{m_{n}}}\left(1+\left(e^{t / \sqrt{m_{n}}}-1\right) e^{-n r_{n}}\right)^{n} \\
& =e^{-t \sqrt{m_{n}}} e^{t \sqrt{m_{n}}+t^{2} / 2+o\left(\frac{1}{n}\right)} \rightarrow e^{-t^{2} / 2}
\end{aligned}
$$


We now turn to asymptotics for the connectivity and largest nearest neighbour distances. Let $c_{n}^{*}, d_{n}^{*}$ respectively, be the connectivity and largest nearest neighbour distances for the graph $G_{n}^{*}$.

Theorem 3. (i) $\lim _{n \rightarrow \infty} \frac{n c_{n}}{\log n}=1, \quad$ a.s.

(ii) $\lim _{n \rightarrow \infty} \frac{n d_{n}}{\log n}=\frac{1}{2}$, a.s.

Proof. (i) To obtain an upper bound, consider the subsequence $n_{k}=k^{a}, a>0$. Then,

$$
\operatorname{Pr}\left(\bigcup_{n=n_{k}}^{n_{k+1}}\left(c_{n} \geq \frac{(1+\epsilon) \log n}{n}\right)\right) \leq \operatorname{Pr}\left(c_{n_{k}} \geq \frac{(1+\epsilon) \log n_{k}}{n_{k+1}}\right) .
$$

Observe that the event $\left\{c_{n} \geq y\right\}=\left\{G_{n}(y)\right.$ is connected $\}$, and hence is a monotone decreasing property. We need to bound the probabilities on the right hand side of (2) by a summable sequence. From lemma 1, this will follow if we can find a summable bound for $\operatorname{Pr}\left(c_{n_{k}}^{*} \geq\left[(1+\epsilon) \log n_{k}\right] /\left(n_{k+1}\right)\right)$. Since

$$
\begin{aligned}
& \operatorname{Pr}\left(c_{n}^{*} \geq y\right)=\operatorname{Pr}\left(\bigcup_{i=1}^{n-1}\left(Y_{n i}>y\right)\right) \leq n e^{-n y} \\
& \operatorname{Pr}\left(c_{n_{k}}^{*} \geq \frac{(1+\epsilon) \log n_{k}}{n_{k+1}}\right) \leq n_{k} \exp \left(-\frac{n_{k}(1+\epsilon) \log n_{k}}{n_{k+1}}\right)=O\left(\frac{1}{k^{\epsilon a}}\right) .
\end{aligned}
$$

Thus we can choose $a$ large enough such that $\operatorname{Pr}\left(c_{n_{k}}^{*} \geq\left[(1+\epsilon) \log n_{k}\right] / n_{k+1}\right)$ is summable and hence

$$
\sum_{k=1}^{\infty} \operatorname{Pr}\left(\bigcup_{n=n_{k}}^{n_{k+1}}\left(c_{n} \geq \frac{(1+\epsilon) \log n}{n}\right)\right)<\infty .
$$

By the Borel-Cantelli Lemma,

$$
\operatorname{Pr}\left(\bigcup_{n=n_{k}}^{n_{k+1}}\left(c_{n} \geq \frac{(1+\epsilon) \log n}{n}\right) \quad \text { i.o. }\right)=0,
$$

and since $\epsilon>0$ is arbitrary, we get

$$
\limsup _{n \rightarrow \infty} \frac{n c_{n}}{\log n} \leq 1, \quad \text { a.s. }
$$

To establish the lower bound, observe that

$$
\operatorname{Pr}\left(c_{n}^{*}<\frac{(1-\epsilon) \log n}{n}\right)=\operatorname{Pr}\left(\bigcap_{i=1}^{n-1}\left(Y_{n i}<\frac{(1-\epsilon) \log n}{n}\right)\right)=O\left(e^{n^{-\epsilon}}\right),
$$

which is summable. Hence by the Borel-Cantelli Lemma, since $\epsilon$ is arbitrary, we get

$$
\lim \inf \frac{n c_{n}}{\log n} \geq 1, \quad \text { a.s. }
$$

This together with (3) completes the proof of (i). 
(ii) Proceeding as in the proof of (i), let $n_{k}=k^{a}$.

$$
\operatorname{Pr}\left(\bigcup_{n=n_{k}}^{n_{k+1}}\left(d_{n} \geq \frac{(1+\epsilon) \log n}{2 n}\right)\right) \leq \operatorname{Pr}\left(d_{n_{k}} \geq \frac{(1+\epsilon) \log n_{k}}{2 n_{k+1}}\right)
$$

Again, $\sum_{k=1}^{\infty} \operatorname{Pr}\left(d_{n_{k}} \geq\left[(1+\epsilon) \log n_{k}\right] /\left(2 n_{k+1}\right)\right)<\infty$, if the same is true for $d_{n}^{*}$. Now,

$$
\begin{aligned}
\operatorname{Pr}\left(d_{n}^{*} \geq t\right) & =\operatorname{Pr}\left(\bigcup_{i=2}^{n-1}\left(\left(Y_{i-1}>t\right) \cap\left(Y_{i}>t\right)\right) \cup\left(Y_{1}>t\right) \cup\left(Y_{n-1}>t\right)\right) \\
& \leq \sum_{i=2}^{n-1} \operatorname{Pr}\left(Y_{i-1}>t\right) \operatorname{Pr}\left(Y_{i}>t\right)+\operatorname{Pr}\left(Y_{1}>t\right)+\operatorname{Pr}\left(Y_{n-1}>t\right) \\
& \leq n e^{-2 n t}+2 e^{-n t} .
\end{aligned}
$$

Hence,

$$
\begin{aligned}
\operatorname{Pr}\left(d_{n_{k}}^{*} \geq \frac{(1+\epsilon) \log n_{k}}{2 n_{k+1}}\right) \leq & n_{k} \exp \left(-\frac{2 n_{k}(1+\epsilon) \log n_{k}}{2 n_{k+1}}\right) \\
& +2 \exp \left(-\frac{n_{k}(1+\epsilon) \log n_{k}}{2 n_{k+1}}\right) \\
\approx & (1 / k)^{\epsilon a} .
\end{aligned}
$$

We can choose $a$ such that $\epsilon a>1$, and hence $\sum_{k=1}^{\infty} \operatorname{Pr}\left(d_{n_{k}}^{*} \geq\left[(1+\epsilon) \log n_{k}\right] /\right.$ $\left.\left(2 n_{k+1}\right)\right)<\infty$. Hence, by the Borel-Cantelli Lemma,

$$
\operatorname{Pr}\left(\bigcup_{n=n_{k}}^{n_{k+1}}\left(d_{n} \geq \frac{(1+\epsilon) \log n}{2 n}\right), \quad \text { i.o. }\right)=0 .
$$

Since $\epsilon$ is arbitrary, we conclude that

$$
\limsup _{n \rightarrow \infty}\left(n d_{n} / \log n\right) \leq 1 / 2 \text {. }
$$

We now establish the lower bound for $d_{n}$. Since,

$$
\begin{aligned}
\operatorname{Pr}\left(d_{n}^{*} \leq t\right)= & \operatorname{Pr}\left(\bigcap_{i=2}^{n-1}\left(\left(Y_{i-1}<t\right) \cup\left(Y_{i}<t\right)\right) \cap\left(Y_{1}<t\right) \cap\left(Y_{n-1}<t\right)\right) \\
= & \prod_{i=2}^{n-1}\left(\operatorname{Pr}\left(Y_{i-1}<t\right)+\operatorname{Pr}\left(Y_{i}<t\right)\right. \\
& \left.-\operatorname{Pr}\left(Y_{i-1}<t\right) \operatorname{Pr}\left(Y_{i}<t\right)\right) \operatorname{Pr}\left(Y_{n-1}<t\right) \operatorname{Pr}\left(Y_{1}<t\right) \\
= & \left(1-e^{-2 n t}\right)^{n-1}\left(1-e^{-n t}\right),
\end{aligned}
$$




$$
\begin{aligned}
\operatorname{Pr}\left(d_{n}^{*} \leq \frac{(1-\epsilon) \log n}{2 n}\right) & =\left(1-e^{-(1-\epsilon) \log n}\right)^{(n-1)}\left(1-e^{-((1-\epsilon) \log n) / 2}\right) \\
& \approx e^{-n^{\epsilon}}, \quad \text { for large } n
\end{aligned}
$$

Hence,

$$
\sum_{n=0}^{\infty} d_{n} \leq[(1-\epsilon) \log n] / 2 n<\infty,
$$

and by the Borel Cantelli Lemma,

$$
\operatorname{Pr}\left(d_{n} \leq[(1-\epsilon) \log n] / 2 n \quad \text { i.o. }\right)=0 .
$$

This gives the lower bound,

$$
\liminf _{n \rightarrow \infty}\left(n d_{n} / \log n\right) \geq 1 / 2, \quad \text { a.s. }
$$

Together with the upper bound we get part (ii) of the theorem.

\section{Graphs in higher dimensions: Asymptotic analyses}

In this section we consider nodes dispersed in $d$ dimensions, $d>1$. Once again, we assume that $w_{i, j}=r$ for all $i, j$. While we could obtain some finite graph results for $d=1$ using combinatorial arguments, such an analysis for higher dimensional graphs are hard and we do not have any such results.

\subsection{Basic definitions}

Let $X_{1}, X_{2}, \ldots$, be a sequence of i.i.d. random variables taking values in $R^{d}, d \geq 2$, according to a bounded probability density function $f$. These represent the node locations in the wireless network. We consider general densities since many of the results for the uniform RGG carry over to the case of non-uniform densities with bounded support without much effort. Asymptotic results, for example, for the maximum vertex degree, chromaticity etc. that are essentially determined by the behavior of the vertices near the mode of the density hold good for nice densities with unbounded supports as well. However, asymptotic results for the minimum vertex degree, the largest nearest neighbour distance, the connectivity distance etc. that are determined by the behaviour of vertices at the tail of the densities, are stated in general for densities with bounded support only. In the latter case the results are characterised by the essential infimum of the density. We mention here that Penrose (2003, Ch. 8) derives asymptotic results for the largest nearest neighbour link for a RGG on normally distributed points.

As before, we consider the $n$-node RGG $G_{n}(r)$ with vertex set $V_{n}=\left\{X_{1}, \ldots, X_{n}\right\}$ and edge set $E_{n}=\left\{\left(X_{i}, X_{j}\right), i, j=1, \ldots, n, i \neq j:\left\|X_{i}-X_{j}\right\|<r\right\}$. Analogous to our use of $G_{n}^{*}$ (RGG generated by independent exponential spacing of the nodes) in studying $G_{n}$ (RGG generated by uniform distribution of nodes in $[0,1])$, the graph $G_{n}(r)$ can be studied using the graph $P_{n}(r)$ with vertex set $\left\{X_{1}, \ldots, X_{N_{n}}\right\}$ and cutoff $r$, where $N_{n}$ is a Poisson random variable with mean $n$. Observe that the graph $G_{n}$ and $P_{n}$ are coupled, in the sense that the first $\min \left(n, N_{n}\right)$ vertices of both graphs are the same. The advantage in working with $P_{n}$ is 
that it is a Poisson point process with intensity $n f(\cdot)$ (proposition 1.5, Penrose 2003). Thus, in the graph $P_{n}$, degrees of two vertices at a distance of more than $2 r$ from each other will be independent. The Palm theory for Poisson point processes (theorem 1.6, Penrose 2003) makes computations of various expectations very easy. Let $h(Y, G)$ be a functional defined for the graph $G$, with $Y \subset G$ satisfying $h(Y, G)=0$, unless $Y$ has $j$ elements. Then

$$
\mathrm{E}\left(\sum_{Y \subset P_{n}} h\left(Y, P_{n}\right)\right)=\left(n^{j} / j !\right) \mathrm{E}\left(h\left(Y_{j}, Y_{j} \cup P_{n}\right)\right),
$$

where $Y_{j}=\left\{X_{1}^{\prime}, \ldots, X_{j}^{\prime}\right\}$ are i.i.d. random variables with pdf $f$. For example, the expected number of triangles (complete subgraphs on 3 vertices) in $P_{n}(r)$ will be $n^{3} / 6$ times the probability that three independent nodes distributed according to $f$ form a triangle at cutoff $r$.

Since we shall obtain only asymptotic results, we need to define the limiting regimes. As in the case of the one-dimensional network, the cutoff $r$ should be a function of $n$ and for the $n$-node network, it is denoted by $r_{n}$. Typically, two limiting regimes are of interest. One is the 'thermodynamic limit' in which $n r_{n}^{d} \rightarrow$ constant. In this regime, the limiting sum of the 'covered volumes' (which is proportional to $r_{n}^{d}$ ) is a constant. This limit is useful in the context of sensor networks where we are interested in the coverage provided. The cases $n r_{n}^{d} \rightarrow 0$ and $n r_{n}^{d} \rightarrow \infty$ are referred to as the sparse and dense limit respectively. The other limiting regime of interest is the connectivity regime, where $n r_{n}^{d} \sim c \log n$. Recall that for one-dimensional networks, we showed that $(\log n) / n$ is a strong threshold for connectivity. This can be extended to show that for a $d$-dimensional network, $((\log n) / n)^{1 / d}$ is a threshold for connectivity of the network. Thus in this limiting regime, the network is asymptotically connected. In this paper, we focus on the connectivity regime. For results under the thermodynamic limits, we refer the reader to Penrose (2003).

We first prove convergence results for the graph $P_{n}$ and then extend them to the graph $G_{n}$ using a technique referred to as 'de-Poissonisation.' Observe that the graph $P_{n}$, on average has the same vertices as the graph $G_{n}$, but there is an additional variance that shows up in the parameter of the limiting distribution. For $\gamma>1 / 2$, and $n$ large enough, a simple application of the Markov inequality for $\exp (z N)$ shows that $\operatorname{Pr}\left(\left|N_{n}-n\right|>n^{\gamma}\right)<c \exp \left(-n^{2 \gamma-1}\right)$ which is summable and hence the events $\left\{\left|N_{n}-n\right|>n^{\gamma}\right\}$ happen only finitely often, a.s. Thus we can restrict attention to the event where $N_{n} \in\left(n-n^{\gamma}, n+n^{\gamma}\right)$. Over this range of $N_{n}$, we need to estimate the change in the value of the functional of interest. This is made more precise in the following theorem. Let $H$ be the functional of interest and let $R_{m, n}=H\left(G_{m+1}\left(r_{n}\right)\right)-H\left(G_{m}\left(r_{n}\right)\right)$. Thus $R$ is the change in the functional $H$ when the number of points in the graph is increased by one.

Theorem 4 (Theorem 2.12, Penrose 2003). Suppose that $n^{-1} \operatorname{Var}\left(H\left(P_{n}\left(r_{n}\right)\right)\right) \rightarrow \sigma^{2}>0$, and

$$
n^{-1 / 2}\left(H\left(P_{n}\left(r_{n}\right)\right)-\mathrm{E}\left(H\left(P_{n}\left(r_{n}\right)\right)\right)\right) \stackrel{d}{\rightarrow} N\left(0, \sigma^{2}\right) .
$$

Let $\alpha$ and $\gamma>1 / 2$ be constants such that the increments $R_{m, n}$ satisfy,

$$
\begin{aligned}
& \lim _{n \rightarrow \infty} \sup _{n-n^{\gamma} \leq m \leq n+n^{\gamma}}\left|\mathrm{E}\left(R_{m, n}\right)-\alpha\right|=0, \\
& \lim _{n \rightarrow \infty} \sup _{n-n^{\gamma} \leq m \leq m^{\prime} \leq n+n^{\gamma}}\left|\mathrm{E}\left(R_{m, n} R_{m^{\prime}, n}\right)-\alpha^{2}\right|=0, \\
& \lim _{n \rightarrow \infty} n^{-1 / 2} \sup _{n-n^{\gamma} \leq m \leq n+n^{\gamma}} \mid \mathrm{E}\left(R_{m, n}^{2}\right)=0 .
\end{aligned}
$$




$$
\begin{aligned}
& \text { If } H\left(G_{m}\left(r_{n}\right)\right) \leq \beta(m+n)^{\beta} \text {, a.s. for some } \beta>0 \text { then } \alpha^{2} \leq \sigma^{2} \text {, and } n^{-1} \operatorname{Var}\left(H\left(G_{n}\left(r_{n}\right)\right)\right) \rightarrow \\
& \sigma^{2}-\alpha^{2} \text { and } \\
& \qquad n^{-1 / 2}\left(H\left(G_{n}\left(r_{n}\right)\right)-\mathrm{E}\left(H\left(G_{n}\left(r_{n}\right)\right)\right)\right) \stackrel{d}{\rightarrow} N\left(0, \sigma^{2}\right) .
\end{aligned}
$$

If we have weak convergence to normality for some functional $H$ for the graph $P_{n}$, then the result will hold for the graph $G_{n}$ provided the value of the functional grows like a polynomial. The expected change in the value of $H$ when one node is added in the $n^{\gamma}$ neighbourhood of $n$ converges to $\alpha$. The asymptotic variance for the normalised $H\left(G_{n}\right)$ is then less than that of $H\left(P_{n}\right)$ by an amount $\alpha^{2}$. We refer the reader to Penrose (2003), lemmas 2.13, 2.14 for sufficient conditions under which the conditions of the above theorem hold.

We are now ready to use the above theorem in our analysis of RGGs in higher dimensions.

\subsection{Subgraphs and component counts}

Let $\Gamma$ be some fixed connected graph on $k$ vertices. $\Gamma$ is said to be feasible if $\operatorname{Pr}\left(G_{k}(r) \cong \Gamma\right)>$ 0 , where $\cong$ indicates that the two graphs are isomorphic. We state a weak convergence result (Theorem 3.12, Penrose 2003) in the connectivity regime, that is when $r_{n}^{d}=(\log n) / n$.

Let $S_{n}(\Gamma)$ be the number of subgraphs in $G_{n}\left(r_{n}\right)$ that are isomorphic to $\Gamma$ and let $h_{\Gamma}\left(x_{1}, \ldots, x_{k}\right)=I_{\left\{G_{k}(1) \cong \Gamma\right\}}$, be the indicator function that the graph $G_{k}(1)$ with vertex set $x_{1}, \ldots, x_{k}$ and cutoff 1 is isomorphic to $\Gamma$. Define,

$$
\mu_{\Gamma}=(k !)^{-1} \int_{R^{d}} f(x)^{k} \mathrm{~d} x \int_{\left(R^{d}\right)^{k-1}} h_{\Gamma}\left(0, x_{1}, \ldots, x_{k-1}\right) \mathrm{d} x_{1} \ldots \mathrm{d} x_{k-1} .
$$

The inner integral is the volume of the set of all configurations of a graph with $k$ vertices with one vertex at the origin, such that the graph formed with maximum allowable edge distance 1 is isomorphic to $\Gamma$.

Theorem 5. Let $\Gamma$ be a feasible connected graph on $k$ vertices and let $r_{n}^{d}=(\log n) / n$. Then

$$
\left(n^{k} r_{n}^{d(k-1)}\right)^{-\frac{1}{2}}\left(S_{n}(\Gamma)-\mathrm{E}\left(S_{n}(\Gamma)\right)\right) \stackrel{d}{\rightarrow} N\left(0, \sigma^{2}=\alpha^{2}\right)
$$

where

$$
n^{k} r_{n}^{d(k-1)} \mathrm{E}\left(S_{n}(\Gamma)\right) \rightarrow \mu_{\Gamma},
$$

and

$$
\begin{aligned}
\sigma^{2}= & (k !)^{-2} \int_{R^{d}} f(x)^{2 k-1} d x \\
& \times \int_{\left(R^{d}\right)^{2 k-2}} h_{\Gamma}\left(0, x_{1}, \ldots, x_{k-1}\right) h_{\Gamma}\left(0, x_{k}, \ldots, x_{2 k-2}\right) d x_{1} \ldots d x_{2 k-2}-\left(k \mu_{\Gamma}\right)^{2} .
\end{aligned}
$$

The result is first proved for the graph $P_{n}$ and then extended to the graph $G_{n}$ using theorem 4 . The first factor in the expression for $\sigma^{2}$ is the asymptotic variance for the Poisson graph. The factor $\alpha$ appearing in theorem 4 in this case is given by $k \mu_{\Gamma}$. 
To see an application of the above theorem, consider $\Gamma$ to be $K_{3}$, the complete graph on 3 vertices. Theorem 4 gives asymptotics in the connectivity regime for the number of triangles in the graph $G_{n}$. The number of triangles in a graph $G$ is a measure of the number of vertices that can communicate with a neighbouring vertex via two paths, one a direct path between the two vertices and the other a two-hop path through another neighbouring vertex. The above theorem thus gives estimates of the number of alternate routes that may be available for communication and thus has a bearing on the total achievable throughput.

\subsection{Maximum vertex degree, chromatic number}

We now discuss the strong law results derived by Appel \& Russo (1997) for the uniform RGG on $[0,1]^{d}$. The results in this section can be extended to more general distributions and arbitrary norms (see chapters 6 and 7, Penrose 2003).

Let $U_{1}, U_{2}, \ldots$, be a sequence of i.i.d. uniform points on $[0,1]^{d}$. The graph $G_{n}(r)$ has vertex set $\left\{U_{1}, \ldots, U_{n}\right\}$ and cutoff $r$. Probability that there is a edge between two nodes $U_{i}$ and $U_{j}$ is given by $p(r)=\operatorname{Pr}\left(\left\|U_{i}-U_{j}\right\| \leq r\right)=\left(2 r-r^{2}\right)^{d}$. Note that $p(r) \approx(2 r)^{d}$ as $r \downarrow 0$.

If $X \sim \operatorname{Bin}(n, p)$, then the Chernoff bounds for $X$ can be written as

$$
\operatorname{Pr}(X \geq k) \leq \exp \left(n p H\left(\frac{n p}{k}\right)\right), \quad k \geq n p,
$$

and

$$
\operatorname{Pr}(X \leq k) \leq \exp \left(n p H\left(\frac{n p}{k}\right)\right), \quad k \leq n p,
$$

where

$$
H(t)=\frac{1}{t} \log t+\frac{1}{t}-1, \quad 0<t<\infty, \quad H(\infty)=-1 .
$$

Note that $H(t)$ is increasing on $(0,1)$, decreasing on $(1, \infty) H(t) \leq 0$, and has a unique maximum $H(1)=0$. The above bounds can be obtained by using the Markov inequality for $\exp (t X), t>0$, and then minimizing w.r.t. $t$.

Let $\epsilon_{n}(r)$ be the total number of edges in $G_{n}(r)$. Then, $\left(\begin{array}{l}n \\ 2\end{array}\right)^{-1} \epsilon_{n}(x)$ is a U-statistics with symmetric kernel $h(u, v)=I_{\{\|u-v\| \leq r\}}, \mathrm{E}\left(\epsilon_{n}(r)\right)=\left(\begin{array}{c}n \\ 2\end{array}\right) p(r)$. Further, since $p(r)$ is a distribution on $[0,1]$, by the Glivenko-Cantelli Lemma, this convergence is uniform. The following proposition gives the rate of convergence in the connectivity regime.

\section{PROPOSITION 1.}

Let $r_{n}$ be a sequence of edge distances satisfying $\frac{n r_{n}^{d}}{\log n} \rightarrow c \in(0, \infty]$. Then,

$$
\lim _{n \rightarrow \infty} \frac{1}{r_{n}^{d}}\left|\left(\begin{array}{l}
n \\
2
\end{array}\right)^{-1} \epsilon_{n}\left(r_{n}\right)-p\left(r_{n}\right)\right|=0
$$

To prove proposition 1, observe that $\left([n / 2] /\left(\begin{array}{l}n \\ 2\end{array}\right)\right) \epsilon_{n}(r)$ can be written as an average of $n$ ! dependent binomial( $[n / 2], p(r))$ random variables:

$$
\epsilon_{n}=\frac{1}{[n / 2] 2 !(n-2) !} \sum_{P} \sum_{k=1}^{[n / 2]} h\left(U_{i_{2 k-1}}, U_{i_{2 k}}\right),
$$


where $\sum_{P}$ is the sum over all permutations $\left\{i_{1}, \ldots, i_{n}\right\}$ of $\{1, \ldots, n\}$. Hence

$$
M_{n}=\left\{[n / 2] \epsilon_{n}\right\} /\left(\begin{array}{l}
n \\
2
\end{array}\right)=(n !)^{-1} \sum_{P} \sum_{k=1}^{[n / 2]} h\left(U_{i_{2 k-1}}, U_{i_{2 k}}\right)=(n !)^{-1} \sum_{p \in P} B_{p},
$$

where $B_{p}$ are binomial $([n / 2], p(r))$. Chernoff bounds for $M_{n}$ can now be obtained by applying the Jensen's inequality as below and then the Chernoff bounds for the Binomial given above.

$$
\begin{aligned}
\operatorname{Pr}\left(M_{n}>k\right) & \leq \frac{\mathrm{E}\left(\exp \left(t M_{n}\right)\right)}{\exp (t k)} \\
& \leq(n !)^{-1} \frac{\sum_{p \in P} \mathrm{E}\left(\exp \left(t B_{p}\right)\right)}{\exp (t k)}=\frac{\mathrm{E}\left(\exp \left(t B_{1}\right)\right)}{\exp (t k)} .
\end{aligned}
$$

Now using a subsequence $\left(n_{k}=k^{r}\right)$ argument (see proofs of results in the one-dimensional case), the Chernoff bounds for $M_{n}$ and the Borel-Cantelli lemma, we can show that $\operatorname{Pr}\left(\left(r_{n}^{-d}\left|\left(\begin{array}{l}n \\ 2\end{array}\right)^{-1} \epsilon_{n}\left(r_{n}\right)-p\left(r_{n}\right)\right|>\epsilon\right.\right.$, i.o. $)=0$.

Let $\Delta_{n}$ denote the maximum vertex degree of the graph $G_{n}\left(r_{n}\right)$.

Theorem 6 (Appel \& Russo 1997a). Let $r_{n}$ be a sequence of edge distance satisfying $\left(n r_{n}^{d}\right) /(\log n) \rightarrow c \in(0, \infty)$. Then,

1. $\liminf \left[\Delta_{n}\left(r_{n}\right)\right] / n r_{n}^{d} \geq 2^{d}$, a.s.

2. $\lim \sup \left[\Delta_{n}\left(r_{n}\right)\right] / n r_{n}^{d} \leq a(c) 2^{d}$, a.s.,

where, $a(c)$ is the root in $[1, \infty)$ of

$$
a \ln a-a+1=1 / 2^{d} c .
$$

Proof of the first part follows immediately from the inequality $n \Delta_{n}(r) \geq 2 \epsilon_{n}(r)$ and proposition 1. To establish the upper bound, first consider

$$
\operatorname{Pr}\left(\frac{\Delta_{n}\left(r_{n}\right)}{n r_{n}^{d}} \geq K\right) \approx \operatorname{Pr}\left(\Delta_{n}\left(r_{n}\right) \geq K c \log n\right) \leq n \operatorname{Pr}\left(\operatorname{deg} U_{i} \geq K c \log n\right) .
$$

Use a subsequence argument as in proposition 1 with $n_{k}=k^{r}$, the above inequality and the Chernoff bounds to obtain the bound $O\left(\left(n_{k}\right)^{\eta+1}\right)$, where $\eta=2^{d} c H\left(2^{d} / K\right)$. The required condition on $K$ is obtained from the summability condition $\eta+1<0$.

The above result can be made sharper. The proof of the following result does not use results from U-statistics as in the above case. It is obtained by first deriving the strong law asymptotics for the $k_{n}$ th nearest neighbour distance for a sequence $k_{n}$ satisfying $k_{n} / \log n \rightarrow b \in(0, \infty]$ and $k_{n} / n \rightarrow 0$.

Theorem 7 (Theorem 6.14, Penrose 2003). Suppose that the vertices of $G_{n}$ are the first $n$ points from an infinite sequence of i.i.d. points distributed according to a density $f$ with compact support. Let $\left(n r_{n}\right) /(\log n) \rightarrow c \in(0, \infty)$. Then

$$
\lim _{n \rightarrow \infty}\left[\Delta_{n} / n \theta r_{n}^{d}\right]=f_{\max } a\left(f_{\max } c\right),
$$

a.s. $a(\cdot)$ is as in theorem 6 and $\theta$ above denotes the volume of the unit ball under the relevant norm. 
Thus the strong law asymptotics for the maximum vertex degree is completely characterised by the mode of the density, which is where one would expect maximum concentration of points and hence points with maximum vertex degree.

An important property of graphs is the clique number. The clique number of a graph is the size of the maximal complete subgraph. Under the $\ell_{\infty}$ norm, the clique number is the maximal number of points that occur in any cube of side $2 r$. This is a form of scan statistic, that has been studied extensively. See, for example Glaz et al (2001). Note that in a wireless network, the nodes that belong to a clique cannot transmit simultaneously. A closely related graph property is the chromatic number. The chromatic number of a graph is the smallest number of colours that can be assigned to the vertices such that no two adjacent vertices have the same colour. Note that the nodes in the same clique cannot be allocated the same colour. In the context of wireless networks, the chromatic number decides the minimum number of channels that are required to schedule the transmission of all the nodes at least once in a cyclic manner. In this manner it is a measure of the minimum capacity that a node can achieve in the network. Chromatic number has been studied in this context by Leese \& Hurley (2002).

Let $C_{n}\left(r_{n}\right)$ and $\chi_{n}\left(r_{n}\right)$ respectively denote the clique and chromatic number of the graph $G_{n}\left(r_{n}\right)$.

Theorem 8 (Theorem 6.16, Penrose 2003). Let $f$ have compact support and $\frac{n r_{n}}{\log n} \rightarrow c \in$ $(0, \infty)$. Then

$$
C_{n} / n \theta r_{n}^{d}=\left(f_{\max } / 2^{d}\right) a\left(f_{\max } c / 2^{d}\right), \quad \text { a.s. },
$$

where $a(\cdot)$ is as in theorem 6.

The following inequality can now be used to obtain strong law bounds for the chromatic number.

$$
C_{n} \leq \chi_{n} \leq \Delta_{n}+1
$$

\subsection{Minimum vertex degree}

We now discuss some results on the minimum vertex degree and the nearest neighbour distance for RGGs. First we consider the RGG on uniform points in $[0,1]^{d}, d \geq 1$ in the $\ell_{\infty}$ norm. Let $\delta_{n}\left(r_{n}\right)$ denote the minimum vertex degree of the graph $G_{n}\left(r_{n}\right)$ under the above assumptions.

Theorem 9 (Appel \& Russo 1997b). Let $r_{n}$ be a sequence of cutoffs satisfying $\left(n r_{n}^{d}\right) /$ $(\log n) \rightarrow c \in(0, \infty]$. Then the following hold.

1. $\lim \sup _{n \rightarrow \infty}\left[\delta_{n}\left(r_{n}\right) / n r_{n}^{d}\right] \leq 1$ a.s.

2. If $c<1 / 2 d$, then $\operatorname{Pr}\left(\delta_{n}\left(r_{n}\right) \geq 1\right.$ i.o. $)=0$.

3. For $0<a<1$, if $c>1 / 2 d|H(2 / a)|$, where $H$ is defined in (4), then $\liminf _{n \rightarrow \infty}\left[\delta_{n}\left(r_{n}\right) /\right.$ $\left.n r_{n}^{d}\right] \geq a$, a.s.

From part (3) of the above theorem, we conclude that for $c>1 /(2 d), \delta_{n}\left(r_{n}\right) \rightarrow \infty$ a.s. whereas for $c<1 /(2 d) \delta_{n}\left(r_{n}\right)$ will be zero eventually a.s.

The largest nearest neighbour distance is defined as $d_{n}=\inf \left\{r: \delta_{n}(r) \geq 1\right\}$. Essentially, this is the cutoff required so that every node has at least one neighbour, i.e., there are no 
isolated nodes in the network. An immediate corollary of parts (2), (3) of theorem 9 is that for $d \geq 1$,

$$
\lim _{n \rightarrow \infty}\left[n d_{n}^{d} / \log n\right]=1 / 2 d .
$$

In contrast to the one-dimensional case where the connectivity distance $c_{n}$ satisfied $n c_{n} / \log n \rightarrow 1$, for $d \geq 2$,

$$
c_{n} / d_{n} \rightarrow 1 \text {. }
$$

This implies that for dimensions $d \geq 2$, the uniform RGG is asymptotically connected with a very high probability for cutoffs (maximum allowable edge length) at which there are no isolated nodes in the graph. In one dimension, one can find a sequence of edge lengths for which, a.s. and for all $n$ large, the graph is disconnected and yet has no isolated vertices. To see this, take a sequence of edge lengths $r_{n}$ such that $n r_{n} / \log n \rightarrow c \in(1 / 2,1)$ in theorem 3 .

Penrose (2003) has generalised the above results to densities with bounded support with smooth boundaries and for arbitrary norms. We conclude this discussion with the statement of a weak law for the largest nearest neighbour distance in case of the uniformly distributed points on $[0,1]^{d}$ under the toroidal metric (see theorem 8.3, Penrose 2003).

$$
\operatorname{Pr}\left(n \theta d_{n}-\log n \leq \alpha\right) \rightarrow \exp (-\exp (-\alpha)), \text { as } n \rightarrow \infty, \quad-\infty<\alpha<\infty .
$$

\section{Beyond identical cutoffs and finite distribution areas}

In the previous sections, we considered networks for which the nodes are distributed in a finite area, typically uniformly in a unit cube or ball in $d$ dimensions and all the cutoffs are identical, i.e., $w_{i, j}=r, \forall i, i \in V$. In this section, we present some generalisations that are possible. The first part is a summary of the results of Desai \& Manjunath (2002) where we consider uniform node distributions but different cutoffs for different node pairs. The second part is derived from the work of Iyer et al (2005) where we consider one-dimensional networks with the node locations being drawn from an exponential distribution.

\subsection{Labelled nodes and specified range matrices}

Let the nodes in $V=\left\{v_{1}, v_{2}, \ldots v_{n}\right\}$ be labelled and, as before, be distributed uniformly in the $d$-dimensional cube $U_{d}=\left[0, x_{0}\right]^{d}$. Define $W:=[w(i, j)]$, with $w(i, j)=w(j, i)$ for all $i, j \in V$. We now obtain the probability that in a random realisation, the distance matrix $W$ is satisfied. A suitably specified $W$ corresponds to checking for the appearance of a labelled subgraph in a random wireless network.

This model can be used to determine the probability that a desirable local configuration involving a small number of nodes is realised, e.g., in sensor networks where a small number of nodes with special capabilities like data collection centres for processing the data collected by the sensors or beacon nodes that help in the self organization of the network. The subgraphs specified by $W$ could include

1. A star network with the central node having special capabilities like a satellite uplink and other nodes being within one hop of this node.

2. A set of nodes with relay capabilities, e.g., those that can form cluster heads which can be at the top of a routing hierarchy. The graph representing the connectivity among these nodes may need to be fully connected. 
Note that the requirement that $w(i, j)=w(j, i)$ is not very restrictive as illustrated by the following examples. Let $i$ be a 'small' node with a 'weak' transceiver and $j$ a 'big' node with a 'strong' transceiver. Although, $i$ transmits with a lower power than $j$, it is not unreasonable to expect that $j$ will be able to receive and decode the signals from $i$. The base station and the mobile node of a cellular network is a classic example of this condition. Now if $k$ were a 'weak' node, the upper bound on $w(i, k)$ for $i$ and $k$ to be able to communicate directly with each other could be much smaller than in $w(i, j)$.

We make a simplification in our analysis. To allow for mathematical tractability, we measure distances using the $l_{\infty}$ norm as against the more realistic $l_{2}$ norm. Usually, the asymptotic behaviour under both the norms are very similar, thus justifying the use of $l_{2}$ norm. However, for finite $n$, we need to appropriately use the norm equivalence theorem to convert the $l_{\infty}$ results to bounds under the $l_{2}$ norm.

Let $X_{i}$ denote the location of $i$. The following inequalities are satisfied if and only if $W$ is satisfied in a realisation of the random network.

$$
\left\|X_{i}-X_{j}\right\|_{\infty} \leq w(i, j), i, j=1,2, \ldots n
$$

Let $P_{W}^{(d)}(\boldsymbol{X})$ denote the probability that the inequalities in (5) are satisfied when $n$ nodes are randomly distributed in $d$-dimensional space. It can be shown that

$$
P_{W}^{(d)}(\boldsymbol{X})=\left(P_{W}^{(1)}(\boldsymbol{X})\right)^{d} .
$$

The problem is thus reduced to that of finding $P_{W}^{(1)}(\boldsymbol{X})$, which is essentially that of finding the connectivity of a heterogeneous one-dimensional network. This is obtained along the lines of the derivation of (1) as follows. Observe that there will be $n$ ! possible ways in which $\boldsymbol{X}$ is realised (corresponding to the possible permutations of $X_{i}$ ), each of which is equally probable. Let $\sigma \in \mathcal{S}_{n}\left(\mathcal{S}_{n}\right.$ is the set of all permutation classes) denote a realization of $\boldsymbol{X}$, i.e., the nodes on $[0, z]$ are ordered as $X_{\sigma_{1}}, X_{\sigma_{2}} \ldots X_{\sigma_{n}}$. Let $P_{W \mid \sigma}^{(1)}(\boldsymbol{X})$ denote the probability that $W$ is satisfied given that the permutation of $\boldsymbol{X}$ is is described by $\sigma$. Then

$$
P_{W}^{(1)}(\boldsymbol{X})=\sum_{\sigma}\left\{P_{W \mid \sigma}^{(1)}(\boldsymbol{X})\right\} / n !
$$

The development of $\S 2$ in obtaining (1) can be generalised to evaluate $P_{W \mid \sigma}^{(1)}(\boldsymbol{X})$.

This result can be used to obtain many probabilities of a finite-node graph. For example, we can obtain the probability that a connected graph occurs that satisfies the distance matrix. We can also exploit symmetries in the organisation of the vector $x$. If we are interested in obtaining the probability of a graph with some symmetric structure, e.g., fully connected graphs, star graphs, line graphs, bipartite graphs, etc., then the calculations is significantly simplified and we can obtain the probability of the occurrence of such graphs in a random finite network.

\subsection{A one-dimensional exponential network}

Consider a random deployment of intrusion detection sensors along a border. It is reasonable to expect that more nodes 'fall' near the point of deployment and fewer away from it. The exponential distribution can capture this property.

Let $n$ nodes be randomly deployed in $(0, \infty)$. Let $X_{i}, i=1, \ldots, n$ be the position of node $i$ with $X_{i}$ being drawn from an exponential distribution with parameter $\lambda$. We assume that all 
nodes have a range of $r$, i.e., nodes $i$ and $j$ are connected if $\left|X_{i}-X_{j}\right|<r$. Let $X_{(i)}, i=$ $1, \ldots, n$, be the locations of the ordered nodes with $X_{(i)}<X_{(i+1)}$. Define $Y_{i}:=X_{(i+1)}-X_{(i)}$ to be the distance between the $i$ th and $(i+1)$ th nodes from the origin. It can be easily seen that $Y_{i}$ are independent random variables with probability density function $f_{Y_{i}}(y)$

$$
f_{Y_{i}}(y)=(n-i) \lambda e^{-(n-i) \lambda y}, \quad \text { for } i=1, \ldots, n-1 .
$$

The above key property of exponential random variables allows us to calculate many important properties of the exponential random network. We now discuss some of these properties.

We first obtain the probability that the network is connected. Let $C_{n}(r)$ denote the probability that an $n$-node network with each node having a transmission range is connected. Note that for the network to be connected we must have $Y_{i}=X_{(i+1)}-X_{(i)}<r, \forall i=1,2, \ldots,(n-1)$. Hence,

$$
\begin{aligned}
C_{n}(r) & =\prod_{i=1}^{n-1} \operatorname{Pr}\left(Y_{i}<r\right)=\prod_{i=1}^{n-1}\left(1-e^{-(n-i) \lambda r}\right) \\
& =\prod_{i=1}^{n-1}\left(1-e^{-i \lambda r}\right) .
\end{aligned}
$$

The asymptotics of $C_{n}(r), \lim _{n \rightarrow \infty} C_{n}$ can also be obtained. It can be shown that

$$
\lim _{n \rightarrow \infty} C_{n}=C .
$$

Similar results can also be obtained for when the nodes are drawn from a two-sided exponential density, $(0.5 \lambda) e^{-0.5 \lambda|x|}$. Let $C_{n}^{(D)}$ denote the connectivity probability of the $n$-node network with node locations drawn from double exponential density. Figure 2 plots $C_{n}$ and $C_{n}^{(D)}$ as functions of $n$ for different values of $(\lambda r)$. Observe that both $C_{n}$ and $C_{n}^{(D)}$ converge rapidly to the asymptote. Thus although we do not have a closed form expression for $C$, it can be well approximated by evaluating a few terms of (6).

For an intrusion detection sensor network, it might be useful to know the properties of the span of a connected network. This is an indication of the coverage provided when the network is connected. It can be shown that the span of the connected network, $S_{C}(n, r)$, is given by

$$
\operatorname{Pr}\left(S_{C}(n, r)<s\right)=P_{n}(s, r) / C_{n},
$$

where $P_{n}(s, r)$ satisfies the recursive relation

$$
\begin{aligned}
P_{n}(s, r) & =\int_{0}^{s \wedge r} P_{n-1}\left(s-y_{n-1}, r\right) f_{Y_{n-1}}\left(y_{n-1}\right) \mathrm{d} y_{n-1}, \\
P_{2}(s, r) & =\operatorname{Pr}\left(0<Y_{1}<r, Y_{1}<s\right) \\
& =\int_{0}^{s \wedge r} f_{Y_{1}}\left(y_{1}\right) \mathrm{d} y_{1} .
\end{aligned}
$$

If the network is not connected, a property of interest is the number of components in the network. It can be shown that the probability that there are $k$ components in the network, $\psi_{n}(1, k)$, is obtained by the recursion,

$$
\psi_{n}(j, k)=q_{j} \psi_{n}(j+1, k)+\left(1-q_{j}\right) \psi_{n}(j+1, k-1),
$$



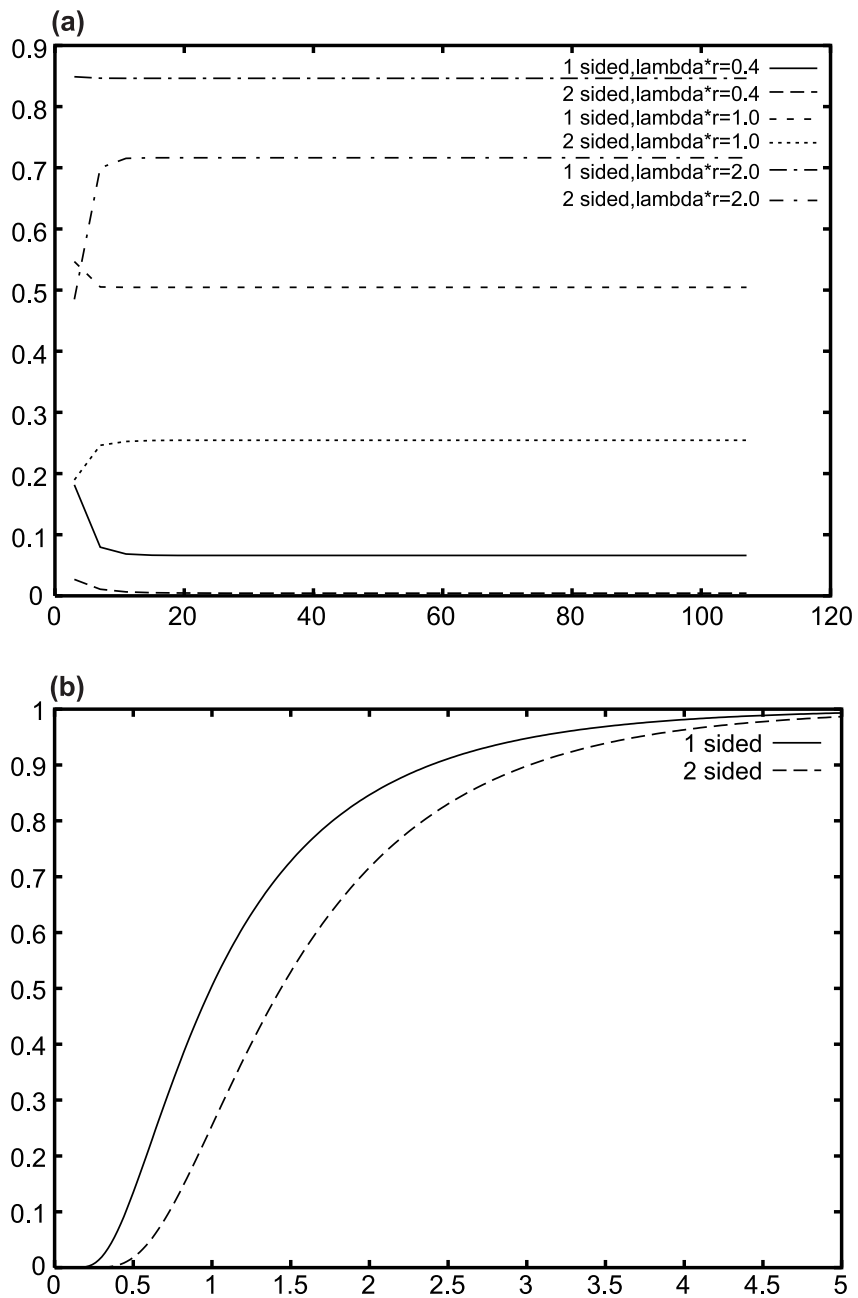

Figure 2. (a) Plot of $C_{n}$ and $C_{n}^{(D)}$ as a function of $n$ for different $r \lambda$. (b) Plot of the asymptotic probability of connectivity in $C$ as a function $r \lambda$.

with initial conditions given by

$$
\begin{gathered}
\psi_{n}(j, n-j+1)=\prod_{i=j}^{n-1}\left(1-q_{i}\right), \\
\psi_{n}(j, 1)=\prod_{i=j}^{n-1} q_{i} .
\end{gathered}
$$

Further, it can be shown that for a given $\lambda r$, the number of components converges in distribution, i.e., probability mass function for the number of components in the network converges as $n \rightarrow \infty$.

In the context intrusion detection networks, we may be interested in the probability that the region $(0, b)$ is covered, i.e., the nodes in this interval will form a connected network. It 
can be shown that this probability is given by

$$
\begin{aligned}
\operatorname{Pr}(\text { nodes in }(0, b) \text { are connected })= & \sum_{m=2}^{n}\left(\begin{array}{l}
n \\
m
\end{array}\right) \exp ^{-\lambda(n-m) b} \\
& \times \prod_{j=1}^{m-1}\left(1-\exp ^{-\lambda j r}\right)\left(1-\exp ^{-\lambda m(b-r(m-1) / 2)}\right) .
\end{aligned}
$$

Consider networks with fixed $\lambda$ and $r$ and $n \rightarrow \infty$. If $Y_{i}>r$, we can say that there is a portion between ordered nodes $i$ and $(i+1)$ that are not covered by the network and that there is a hole of size $Y_{i}-r$. If we think of the nodes as sensors with range $r$ deployed along a border, then an intruder passing through the hole goes undetected. Denoting the length of the hole between the nodes $i$ and $(i+1)$ by $Z_{i}$, we have

$$
Z_{i}=\max \left\{Y_{i}-r, 0\right\} .
$$

The total length of the holes in the network is then $H(n, r):=\sum_{i=1}^{n} Z_{i}$ and the number of holes is $N H(n, r):=\sum_{i=1}^{n} I_{\left\{Y_{i}>r\right\}}$. Note that the number of holes is one plus the number of components. Asymptotic analysis of the number of holes and the total length of the holes gives us the following result.

Theorem 10. As $n \rightarrow \infty$ the $H(n, r)$ and $N H(n, r)$ converge in distribution to a random variable with finite mean and variance.

$$
\begin{gathered}
H(n, r) \stackrel{\text { dist }}{\rightarrow} H(r), \\
N H(n, r) \stackrel{\text { dist }}{\rightarrow} N H(r),
\end{gathered}
$$

where $H(r)$ and $N H(r)$ are the limiting random variables with finite mean and variance.

\section{An annotated bibliography}

The discussion above is only a sample of the literature on this emerging area of random geometric graphs and topology models for wireless networks. In the following we attempt an annotated bibliography, possibly incomplete but sufficiently wide in scope and time, which we believe will be useful to start research in the area. We begin by discussing wireless network literature and then describe the literature on RGGs. It might be mentioned here that the recent book by Penrose (2003) is quite comprehensive in its coverage and is also an excellent source for references for RGGs.

Random multihop wireless networks have been studied ever since the days of the Aloha network in the 1970s. However, much of the early interest had subsided till the 1990s when interest was revived with the development of IEEE 802.11 Wireless LANs and the emerging paradigms of sensor networks.

The connectivity of one-dimensional networks with the nodes being uniformly distributed on a finite segment and all nodes having identical transmission range has been derived by Desai \& Manjunath (2002). Godehardt \& Jaworski (1996) also study properties of similar structures under the name of interval graphs. McColm (2004) obtains threshold functions for such graphs. Iyer et al (2005) obtain many connectivity properties of one-dimensional networks 
in which node locations are assumed i.i.d. exponential. Finite network and asymptotic results are obtained. Karamchandani et al (2005) derive clustering properties of such networks.

For a network of $n$ nodes distributed in a unit circle where every node transmits using omnidirectional antennas and has the same transmission range $r_{n}$, Gupta \& Kumar (1998) obtain the asymptotic threshold function for the critical transmission radius to be $r_{c}(n)=$ $\sqrt{(\log n) / n}$. The transmission radius for connectivity of a network when the placement of the nodes follows a Poisson process in dimensions $d \leq 2$ is derived by Cheng \& Robertazzi (1989). Panchapakesan \& Manjunath (2001) also analyse the connectivity under uniform and Poisson node distribution assumptions. More recently, Santi \& Blough (2003) obtain the transmission radius for sparse networks. Bettstetter \& Hartmann (2005) study the connectivity when the SNR on the links is affected by channel fading.

A result that enables the nodes to control local topological properties, i.e., properties of their neighbourhood, and work towards obtaining a connected network is derived by Xue \& Kumar (2004) where it is shown that for a static network, if each node is connected to $5.1774 \log n$ nodes, the network is asymptotically connected. This problem has also been studied empirically in the context of multi-hop slotted Aloha networks by Kleinrock \& Silvester (1978). This result enabled Borkar \& Manjunath (2005) to devise a control law that could control the topology based only on local topological information. Desai \& Manjunath (2005) consider finite networks in which the transmission ranges are not uniform and obtain some connectivity properties.

An important communication property is the information carrying capacity of a randomly deployed networy. Gupta \& Kumar (2000) derive the maximum possible throughput between a random communicating pair in a random multihop network to be $\Theta\left(1 /(n \log n)^{1 / 2}\right)$. Further it is shown that the throughput is at most $O(1 / \sqrt{n})$ when the nodes are optimally placed rather than randomly deployed. Grossglauser \& Tse (2001) show that if the mobility of the nodes can be exploited then the capacity can be made $O(1)$. The information theory for wireless networks is developed by Gupta \& Kumar (2003) and Xie \& Kumar (2004).

As we mentioned earlier, sensor networks perform specific functions in a distributed manner. These functions would require that the communication patterns be executed. For example, each sensor may want to send its data to every other node in the network using, say, a flooding algorithm. Zuniga \& Krishnamachari (2006) obtain the critical radius necessary to optimally flood the network. Another class of properties is the rate at which specific functions may be computed. Giridhar \& Kumar (2005) studies the maximum rate at which functions of sensor measurements can be computed and communicated to the sink node. Khude et al (2005) study the time and energy complexity of performing distributed functions over random geometric graphs. They also consider pipelining of the functions.

There is a significant amount of literature on the analysis of random geometric graphs. An early survey is by Diaz et al (1998). Appel \& Russo (1997) obtained the asymptotic threshold functions for maximum and minimum vertex degrees for RGGs constructed by distributing $n$ points in a $d$-dimensional cube. They use the $l_{\infty}$ norm. Penrose has obtained many results. He obtains the longest edge of the minimal spanning tree (Penrose 1997). He shows (Penrose 1999) that the threshold function for making the network $k$-connected is the same as the threshold function for which the minimum node degree is $k$. An excellent introduction to the kinds of results and the techniques used in obtaining these results is available in the book by Penrose (2003). In fact much of our discussion in $\S 3$ is aimed at making this book more accessible to the reader. It is also an excellent source for further references on RGGs. Goel et al (1998) show that all monotone properties on RGGs have sharp thresholds and that they are much sharper than that of the Erdos-Renyi graphs. 


\section{References}

Appel M J B, Russo R 1997a The maximum vertex degree of a graph on uniform points in $[0,1]^{d}$. Adv. Appl. Probab. 29: 567-581

Appel M J B, Russo R 1997b The minimum vertex degree of a graph on uniform points in $[0,1]^{d}$. Adv. Appl. Prob. 29: 582-594

Bettstetter C, Hartmann C 2005 Connectivity of wireless multihop networks in a shadow fading environment. ACM/Kluwer Wireless Networks. Special Issue on Modeling and Analysis of Mobile Networks vol. 11

Bollobas B 1985 Random graphs (Academic Press)

Borkar V S, Manjunath D 2005 A distributed control law for incremental power adaptation based topology control of ad hoc wireless networks. In Proceedings of WiOpt-05, Trentino, Italy

Cheng Y C, Robertazzi T 1989 Critical connectivity phenomena in multihop radio models. IEEE Trans. Commun. 37: 770-777

Desai M P, Manjunath D 2002 On the connectivity in finite ad-hoc networks. IEEE Commun. Lett. 6: 237-239

Desai M P, Manjunath D 2005 On range matrices and wirelesss networks in $d$-dimensions. In Proceedings of WiOpt-05, Trentino, Italy

Diaz J, Petit J, Serna M 1998 Random geometric graph in $[0,1]^{2}$. In Randomization and approximation techniques in computer science, Proc. Second Int. Workshop, RANDOM'98 (eds) M Luby, J D P Rolim, M J Serna (Lecture Notes in Computer Science 1518) (Berlin: Springer-Verlag)

Friedgut E, Kalai G 1996 Every monotone graph property has a sharp threshold. Proc. Am. Math. Soc. 124: 2993-3002

Giridhar A, Kumar P R 2005 Computing and communicating functions over sensor networks. IEEE J. Selected Areas Commun. (to appear)

Glaz J, Naus J, Wallenstein S 2001 Scan statistics (Springer)

Godehardt E, Jaworski J 1996 On the connectivity of a random interval graph. Random Struct. Algorithms 9: 137-161

Goel A, Rai S, Krishnamachari B 2004 Sharp thresholds for monotone properties in random geometric graphs. In ACM Symp. on Theory of Computing (STOC)

Grossglauser M, Tse D 2001 Mobility increases the capacity of wireless ad hoc networks. In Proc. of IEEE Infocom, Anchorage, Alaska

Gupta P, Kumar P R 1998 Critical power for asymptotic connectivity in wireless networks. In Stochastic analysis, control, optimization and applications: A volume in honor of W. H. Fleming (eds) W M McEneany, G Yin, Q Zhang (Boston: Birkhauser) pp 547-566

Gupta P, Kumar P R 2000 The capacity of wireless networks. IEEE Trans. Inf. Theor. 46: 388-404

Gupta P, Kumar P R 2003 Towards an information theory of large networks: An achievable rate region. IEEE Trans. Inf. Theor. 49: 1877-1894

Iyer S K, Manjunath D, Gupta B 2005 On the topological properties of one dimensional exponential random geometric graph. (submitted)

Karamchandani N, Manjunath D, Iyer S K 2005 On the clustering properties of exponential random networks. In Proc. IEEE Int. Symp. World of Wireless, Mobile and Multimedia Networks (WoWMoM), Taormina, Italy

Khude N, Kumar A, Karnik A 2005 Time and energy complexity of distributed computation in wirelss sensor networks. In Proc. IEEE Infocom

Kleinrock L, Silvester J A 1978 Optimum transmission radii in packet radio networks or why six is a magic number. In Proc. Natl. Telecommunications Conference, Birmingham, pp 04.3.1-5

Kumar A, Manjunath D, Kuri J 2004 Communication networking: An analytical approach (Morgan Kaufman)

Leese R, Hurley S 2002 Methods and algorithms for radio channel assignment (Oxford: University Press) 
McColm G L 2004 Threshold functions for random graphs on a line segment, Combinatorics, Probab. Comput. 13: 373-387

Panchapakesan P, Manjunath D 2001 On the transmission range in dense ad hoc radio networks. In Proc. SPCOMM 2001

Penrose M D 1997 The longest edge of the random minimal spanning tree. Ann. Appl. Probab. 7: 340361

Penrose M D 1999 On $k$-connectivity for a geometric random graph. Random Struct. Algorithms 15: $145-164$

Penrose M D 2003 Random geometric graphs (Oxford: University Press)

Santi P, Blough D M 2003 The critical transmitting range for connectivity in sparse wireless ad hoc networks. IEEE Trans. Mobile Comput. 2: 25-39

Xie L L, Kumar P R 2004 A network information theory for wireless communication: Scaling laws and optimal operation. IEEE Trans. Inf. Theor. 50: 748-767

Xue F, Kumar P R 2004 The number of neighbours needed for connectivity of wireless networks. Wireless Networks 10: 169-181

Zuniga M, Krishnamachari B 2006 Optimal transmission radius for flooding in large scale sensor networks. J. Cluster Comput. (to appear) 